\title{
Gastronomía típica como identidad cultural
}

\author{
Typical gastronomy as a cultural identity
}

1 Karina Patricia Llerena Oñate https://orcid.org/0000-0002-0443-1436 Instituto Superior Tecnológico Tungurahua, Carrera de Tecnología Superior en Gastronomía, Ambato, Ecuador.

kllerena@institutos.gob.ec

2 Daniel Oswaldo Sánchez Guerrero https://orcid.org/0000-0003-3104-9125 Universidad Técnica de Ambato, Facultad de Ciencias Humanas y de la Educación, Carrera de Turismo. Ambato, Ecuador. do.sanchez@uta.edu.ec

$3 \quad$ Vanessa Catalina Lizano Saltos https://orcid.org/0000-0002-0652-7431 Instituto Superior Tecnológico Tungurahua, Carrera de Tecnología Superior en Gastronomía, Ambato, Ecuador. vlizano@institutos.gob.ec

4 Dayana Micaela Ruíz Villegas https://orcid.org/0000-0002-6392-306X Universidad Técnica de Ambato, Facultad de Ciencias Humanas y de la Educación, Carrera de Turismo. Ambato, Ecuador. druiz3457@uta.edu.ec

Artículo de Investigación Científica y Tecnológica Enviado: 24/12/2021

Revisado: $29 / 12 / 2021$

Aceptado: $12 / 01 / 2022$

Publicado:08/03/2023

DOI: https://doi.org/10.33262/concienciadigital.v6i1.4.2000

CONCIENCIA DIGITAL, es una Revista Multidisciplinar, Trimestral, que se publicará en soporte electrónico tiene como misión contribuir a la formación de profesionales competentes con visión humanística y crítica que sean capaces de exponer sus resultados investigativos y científicos en la misma medida que se promueva mediante su intervención cambios positivos en la sociedad. https://concienciadigital.org

La revista es editada por la Editorial Ciencia Digital (Editorial de prestigio registrada en la Cámara Ecuatoriana de Libro con No de Afiliación 663) www.celibro.org.ec 
Palabras claves:

gastronomía, identidad cultural, Pelileo, restaurantes, turismo, Tungurahua.

\section{Keywords:}

cultural

identity, gastronomy, Pelileo, restaurant, tourism, Tungurahua

\section{Resumen}

Introducción. Actualmente la gastronomía típica es considerada como un patrimonio importante para el desarrollo del turismo, la degustación de platos típicos genera una experiencia cultural y sensorial donde los comensales tienen una conexión con la gastronomía de la zona. Objetivo. Analizar la gastronomía típica como identidad cultural de los habitantes de Pelileo. Metodología. La investigación tuvo un diseño mixto, es decir, cuantitativo utilizando un cuestionario como herramienta y cualitativo utilizando los formularios de registro del Instituto Nacional de Patrimonio Cultural (INPC) con un estudio de campo. El cuestionario fue enviado a 383 pobladores de Pelileo, el cuestionario fue elaborado en la plataforma de formularios de Google y se realizó un análisis de confiabilidad para su aplicación. El levantamiento de información sobre la gastronomía típica en 179 establecimientos de Pelileo se realizó mediante fichas de registro (INPC), campo de estudio que es tan descriptivo como deductivo. Gracias a la ficha de registro (INPC), se identificaron los platos típicos más típicos del cantón como son: cuy asado, conejo asado, fritada, empanadas de dulce, hornado, tamales, chawarmisqui, caldo de gallina y empanadas de viento. Resultados. Entre los resultados de la encuesta, cabe señalar que la mayoría de los habitantes del cantón muestran una inclinación sobre la dimensión efectiva con una media de 3.80 correspondiente al $19.84 \%$ Conclusiones. Las emociones se superponen a otros aspectos más racionales, se requiere por tanto un mayor grado de relevancia para informar por los medios posibles la comida típicamente representativos.

\section{Abstract}

Introduction. Currently the typical gastronomy is considered an important heritage for the development of tourism, the tasting of typical dishes generates a cultural and sensorial experience where diners have a connection with the gastronomy of the area. Objective. Analyze the typical gastronomy as a cultural identity of the inhabitants of Pelileo. Methodology. The research had a mixed design, that is, quantitative using a questionnaire as a tool and qualitative using the registration forms of the National Institute of Cultural Heritage (INPC) with a field study. The questionnaire was sent to 383 inhabitants of Pelileo, the questionnaire was prepared 
on the Google forms platform and a reliability analysis was carried out for its application. The collection of information on typical gastronomy in 179 establishments in Pelileo was carried out using registration cards (INPC), a field of study that is as descriptive as it is deductive. Thanks to the registration form (INPC), the most typical dishes of the canton were identified, such as: roast guinea pig, roast rabbit, fried, sweet empanadas, baked, tamales, chawarmisqui, chicken broth and wind empanadas. Results. Among the results of the survey, it should be noted that most of the inhabitants of the canton show an inclination on the effective dimension with an average of 3.80 corresponding to $19.84 \%$. Conclusions. Emotions are superimposed on other more rational aspects; therefore, a greater degree of relevance is required to inform the typically representative food by the possible means.

\section{Introducción}

La gastronomía típica es muy valorada por el turista que visita los establecimientos de alimentos y bebidas conocidos por sus únicas características. Por esta razón es preciso que los restaurantes que ofertan comida típica tengan buena presentación y brinden un servicio adecuado, sin perder sus rasgos naturales. Los establecimientos de alimentación tendrán la oportunidad de formar un atractivo turístico que el visitante siempre deseará volver a experimentarlo (Mogollón et al., 2015).

En la Revista Amazónica Ciencia y Tecnología, se publicó una investigación con el tema: "La gastronomía como recurso de la identidad ancestral: el caso de la parroquia Chuquiribamba, Loja, Ecuador", en donde se identificó nueve elementos, los ingredientes para usar en la elaboración de platillos son productos agrícolas, de la misma forma se procedió a una investigación participativa que ha permitido valorar el patrimonio cultural inmaterial, rescatar la memoria de los pobladores y promocionar los recursos gastronómicos a los turistas interesados en visitar la parroquia (Fernández y Miret, 2019).

Cuando el hombre cultivo la tierra, apareció el maíz, cebada y trigo procedente de Asia Menor, se consumía la mayoría de los productos en su estado natural. La cocina se basa generalmente en la búsqueda de la supervivencia, el descubrimiento del fuego fue un punto de partida de evolución, el ser humano paso de comer carne cruda a empezar a preparar platos más deliciosos. Los frutos y la carne han sido lavados en agua de mar, donde se ha detectado el sabor a sal, a partir de este punto la comida comienza a mezclarse con especias para obtener diferentes sabores (Bravo y Vallejo, 2018).

La importancia de la gastronomía de cada región del mundo radica en que nos habla de la cultura de cada pueblo al que pertenece y que la gastronomía, además de brindarnos 
deliciosos sabores y platos únicos, indirectamente nos puede decir sobre las costumbres y la forma de vida. Ahora la gastronomía ha jugado un papel indudablemente importante en la experiencia que los visitantes obtienen de un destino turístico, esta es definitivamente una oportunidad con un enorme potencial para negocios como el turismo, la cultura y las industrias culinarias (Bugallo, 2020).

En una investigación sobre: "La gastronomía típica como un factor de desarrollo turístico del cantón Tisaleo provincia de Tungurahua", menciona que la motivación por la gastronomía se da por varias razones que impulsan el interés en los visitantes, como el interactuar con una cultura diferente, sus costumbres y tradiciones culturales del lugar que se está conociendo, por lo tanto la gastronomía típica es un patrimonio principal en el desarrollo turístico y sobre todo económico y cultural del cantón, convirtiéndolo así en un atractivo con productos sanos y nutritivos que influye en la identidad cultural de Tisaleo (Bombón, 2017).

La identidad cultural se define como una serie de tradiciones, valores y costumbres que conforman un determinado grupo o comunidad de personas, a través de las cuales pueden construir un sentido de pertenencia y preservar las características únicas de cada nación. Además, se caracteriza por estar claramente influida de manera notoria por el entorno en el que se desenvuelve un individuo, en relación con el contexto histórico y cultural (Saltos \& Raymond, 2018).

Para mantener la identidad cultural, los individuos necesitan saber por qué la importancia del folclore, las tradiciones, todo a través del conocimiento y la comprensión. La identidad cultural no solo puede estudiarse como un fenómeno aislado, sino que también puede surgir como una oposición a otras identidades, en la figura 2 se pueden observar cuatro tipos de factores de identidad cultural con sus respectivos significados (Bohórquez et al., 2016).

Por otra manera, en la investigación de: "Fortalecimiento histórico cultural de la gastronomía del cantón San Pedro de Pelileo, provincia de Tungurahua”, menciona que a través de su estudio se diseñó una guía gastronomía con características culinarias, innovadoras, acordes a cada preparación y su fácil comprensión. Todo esto ayuda a fortalecer el turismo y por ende la identidad cultural del cantón, sobre todo a los empresarios a mejor sus productos y que se pueda expender comida típica, informándose de la variedad de productos ancestrales existentes que Pelileo ofrece (Moposita, 2016).

Según Navarrete (2015), menciona que la identidad es un concepto muy amplio ya que abarca varios factores, características y hechos haciendo al ser humano único, sin la responsabilidad de que exista otra persona igual, simplemente pueden tener similitudes ya que cada uno tiene sus propias experiencias vivenciales y cualidades propias del mismo. Es importante tener una identidad ya que ayuda a ser fuertes, decididos y 
comprometidos con lo que se quiere, se respeta la individualidad, el aceptarse como es y sobre todo cumplir con el propósito para la vida mientras que la falta de identidad hará que la persona sea mala influenciada por terceras personas causando un daño propio, se puede encontrar diferentes tipos de identidad que lo presentamos a continuación:

Identidad cultural. - Son aquellas características que se enfocan en una determinada cultura, como las creencias, costumbres, tradiciones, valores que posee una determinada comunidad que los hace únicos y diferentes de los demás.

Identidad personal. - Es aquella que posee cada persona al momento de ser asignado nombre y apellido.

Identidad nacional. - Hace referencia a una nación o territorio del cual pertenece en las que incluyen la cultura y la lengua.

Identidad de género. - Son las percepciones subjetivas que un individuo tiene sobre sí mismo, el cual puede o no coincidir con sus características sexuales.

Un modelo de estudio de la identidad cultural, que consta de tres subsistemas que son: proceso de formación axiológica, proceso de gestión extensionista y proceso contextual de identidad de la cultura universal. Garza y Llanes (2015), propone en la figura 3 donde se puede observar el modelo con mayor claridad y el ciclo que cumple:

Proceso de formativo axiológico. - Propia realidad social con relación de significación entre procesos y acontecimientos de la vida social, así como los intereses y necesidades los cuales son dinámicos, cambiantes y dependientes de las condiciones.

Proceso de gestión extensionista. - Procesos de investigación, docencia y extensión favorece al proceso docente para que se desarrolle dentro de la institución y fuera de ella. Diversas actividades formativas en los componentes curriculares

Proceso contextual pedagógico cultural. - El componente identidad social es un proceso en el que la educación y la sociedad mantienen relaciones dialécticas. La identidad social permite a los integrantes de la comunidad adquirir y desarrollar conocimientos y habilidades para analizar y solucionarlo.

Por otra parte, hay 2 corrientes en antropología al momento de abordar el fenómeno de la identidad cultural: la perspectiva esencialista y la constructivista en donde cada una aporta un gran valor (Martínez, 2015).

Perspectiva esencialista. - Se encarga del estudio de los conflictos de identidad como algo inminente y hereditario culturalmente, lo que significa que los diversos rasgos culturales son transmitidos a través de generaciones, configurando una identidad cultural mediante el tiempo. 
Perspectiva constructivista. - Menciona que la identidad no es algo que se hereda sino únicamente algo que se construye, por lo que no es algo estático, solido o inmutable, al contrario, es dinámico, maleable y manipulable.

Se conoce que la identidad cultural no es algo estable-inmutable, sino que puede cambiar, reformularse y verse influenciada por otras culturas. Este intercambio puede darse de manera pacífica a través de relaciones sociales, culturales o comerciales (Lardellier, 2015). Este proceso de cambio de identidad cultural puede llamarse varias formas como:

Aculturación. - cuando se sustituye elementos propios de una cultura por los provenientes de otra ya sea por conquista, invasión, colonización, etc., de esta manera y por diversas causas varios individuos o grupos sociales han modificado o adaptado diversos elementos culturales propios por otros poniendo en peligro la perdida de cultura, lo cual se ha venido dando desde tiempos remotos a lo largo de la historia al mantener contacto con comunidades sociales diferentes.

Transculturación. - un grupo social recibe y adopta las formas culturales que proviene de otros grupos, por lo que termina sustituyendo parcialmente sus propias prácticas sociales. El proceso de transculturación se puede convertir en dolorosa ya que involucra la pérdida de identidad y sumisión frente al otro. Es una cultura que impone sus tradiciones y costumbres en otra, de esta manera termina perdiendo la otra aquello que le caracterizaba o simplemente era diferente frente al mundo como es el caso de la globalización.

Inculturación. - Es la integración de un individuo o grupo en la cultura y en la sociedad con la que entra en contacto.

\section{Metodología}

El lugar donde se realizó la presente investigación se encuentra ubicado en la provincia de Tungurahua, a 153 kilómetros de la ciudad de Quito capital del país. El nombre del cantón es San Pedro de Pelileo y posee una extensión territorial de $202.98 \mathrm{~km}^{2}$ aproximadamente, se encuentra dividida en 8 parroquias rurales como son: García Moreno, Benítez, Cotaló, Huambaló, Salasaca, El Rosario, Bolívar y Chiquicha; una urbana: La Matriz (Pelileo y Pelileo Grande). Pese al desastre natural del terremoto ocurrido el 5 de agosto de 1949 la población demuestra una amplia capacidad para superar las adversidades y buscar nuevos horizontes para salir de las crisis (Pérez et al., 2017). En la figura 1 se detalla la ubicación geográfica del Cantón San Pedro de Pelileo. 


\section{Figura 1}

Ubicación geográfica del cantón Pelileo

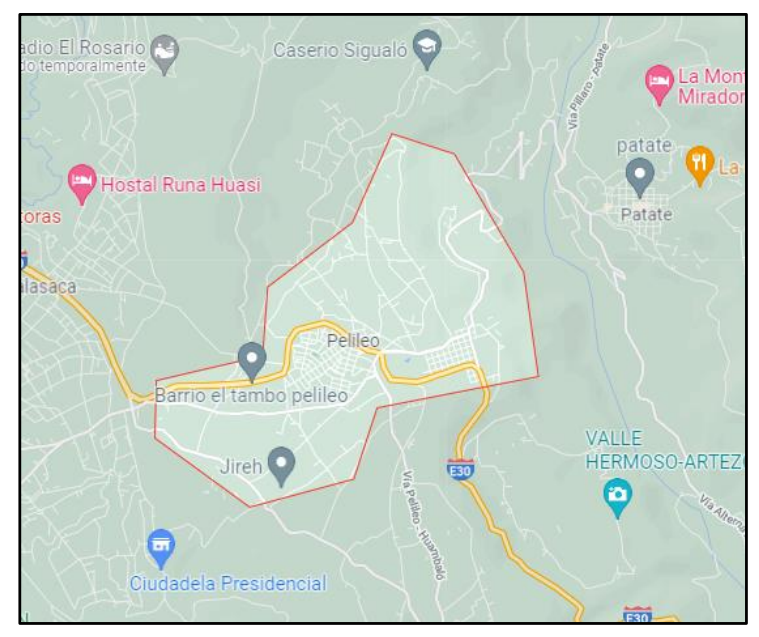

Fuente: Google maps

\section{Tipo de investigación}

Se empleó una metodología mixta, es decir cuantitativa por medio del instrumento como el cuestionario y cualitativa aplicada a la recolección de datos a través de fichas de registro del INPC, el alcance de la investigación fue de tipo transversal descriptivo, es decir un tipo de indagación observacional centrado en analizar datos de una determinada población de muestra en un periodo de tiempo determinado. Además, se enmarca en una lógica deductiva lo cual parte de una premisa general para obtener las conclusiones de un caso particular, poniendo énfasis en la teoría, modelos teóricos, la explicación y abstracción, antes de recoger datos empíricos, observaciones o usar experimentos (Castellanos, 2017).

\section{Población}

Según el catastro de patentes del 2021 del Gobierno Autónomo Descentralizado Municipal de Pelileo (GAD Municipal Pelileo) registra un total de 334 establecimientos que se dedican a la elaboración de comidas típicas. Para el levantamiento de información en los establecimientos se realizó un muestreo aleatorio por conglomerados. Un muestreo por conglomerados es cuando a la población se divide en grupos a partir de los límites naturales geográficos o de otra clase (Lind et al., 2012).

A continuación, se seleccionan los conglomerados al azar y se toma una muestra de forma aleatoria con elementos de cada grupo. El tamaño de la muestra es de 179 establecimientos con un nivel de confianza del $95 \%$. En la Tabla 1 se detalla el número de establecimientos con su correspondiente número de fichas de registro a realizar. 


\section{Tabla 1}

Número de establecimientos del cantón San Pelileo y su respectivo número de fichas de registro

\begin{tabular}{cccc}
\hline Parroquias & Establecimientos & $\%$ & Número de fichas \\
\hline La Matriz & 245 & 73 & 131 \\
Benítez & 12 & 4 & 6 \\
Bolívar & 6 & 2 & 3 \\
Cotaló & 5 & 1 & 3 \\
Chiquicha & 2 & 1 & 1 \\
El Rosario & 2 & 1 & 1 \\
García Moreno & 13 & 4 & 7 \\
Huambaló & 14 & 4 & 8 \\
Salasaca & 35 & 10 & 19 \\
Total & 334 & 100 & 179
\end{tabular}

Fuente: Llerena (2021)

Según los resultados del censo de población y vivienda ejecutados en Ecuador en el año 2010, el cantón de San Pedro de Pelileo registra un total de 56573 habitantes. Para la realización de las encuestas se realizó un cálculo para determinar el tamaño de la muestra. La muestra representativa para el presente estudio es de 382 habitantes. En la Tabla 2 se detalla el número de habitantes de cada parroquia del cantón con su correspondiente número de encuestas a realizar.

\section{Tabla 2}

Número de habitantes por parroquia del cantón San Pedro de Pelileo y su respectivo número de encuestas

\begin{tabular}{cccc}
\hline Parroquias & Habitantes & & \\
& & Porcentaje \% & Número de personas encuestadas \\
\hline La Matriz & 24614 & 44 & 166 \\
Benítez & 2183 & 4 & 15 \\
Bolívar & 2713 & 5 & 18 \\
Cotaló & 1852 & 3 & 13 \\
Chiquicha & 2445 & 4 & 17 \\
El Rosario & 2638 & 5 & 18 \\
García Moreno & 6380 & 11 & 43 \\
Huambaló & 7862 & 14 & 53 \\
Salasaca & 5886 & 10 & 40 \\
TOTAL & 56573 & 100 & 383
\end{tabular}

Fuente: Llerena (2021) 


\section{Muestra}

Para calcular el tamaño de muestra de los habitantes por parroquia del cantón San Pedro de Pelileo adecuada se utilizó la ecuación [1] que se detalla a continuación.

$$
\mathrm{n}=\frac{\mathrm{N} * \mathrm{Z}_{\propto}^{2} * \mathrm{p} * \mathrm{q}}{\mathrm{e}^{2} *(\mathrm{~N}-1)+\mathrm{Z}_{\propto}^{2} * \mathrm{p} * \mathrm{q}}
$$

\section{Ecuación 1}

Donde:

$\mathrm{n}=$ tamaño de muestra buscado.

$\mathrm{N}=$ total de la población.

Z_ $\propto^{\wedge} 2=$ Parámetro estadístico y depende del nivel de confianza (nivel seguridad $=95 \%$ ). $\mathrm{p}=$ probabilidad que ocurra el evento deseado (probabilidad de éxito; si se desconoce es igual a 0,5$)$.

$\mathrm{q}=1-\mathrm{p}$; probabilidad que no ocurra el evento deseado.

e $=$ error de estimación máximo aceptado (para este tipo de investigación se asume un error del $5 \%$ ).

$$
\begin{gathered}
\mathrm{n}=\frac{\mathrm{N} * \mathrm{Z}_{\propto}^{2} * \mathrm{p} * \mathrm{q}}{\mathrm{e}^{2} *(\mathrm{~N}-1)+\mathrm{Z}_{\propto}^{2} * \mathrm{p} * \mathrm{q}} \\
\mathrm{n}=\frac{56573 *(1,96)^{2} * 0,5 * 0,5}{0,05^{2} *(56573-1)+(1,96)^{2} * 0,5 * 0,5} \\
\mathrm{n}=383
\end{gathered}
$$

\section{Recolección de información}

\section{Técnicas e instrumentos de investigación}

La investigación utilizó una encuesta como técnica de recolección de datos, mediante el cuestionario y se obtuvo información relacionada con la caracterización de la identidad cultural del habitante de Pelileo a causa de la gastronomía típica. Además, se usó la ficha A4 INPC para identificar la gastronomía típica a través de un estudio de campo, dirigido a los establecimientos de alimentos y bebidas que constan en el catastro.

Análisis de fiabilidad de la escala de confianza

Se aplicó la prueba Alfa de Cronbash para comprobar la fiabilidad del instrumento de investigación en donde se emitió un valor de 0,811 , el mismo que se considera un valor aceptable para su aplicación, como podemos observar en la Tabla 3 se detalla la prueba 
de Chi Cuadrado y en Tabla 4 se detalla las dimensiones de diseño de un instrumento para medir identidad cultural indígena, caso de estudio sobre la nacionalidad Amazónica.

Según la Tabla 3 para la prueba de hipótesis se empleó el método chi-cuadrado, donde se observa que la significación asintótica (bilateral) es $0,000<0,05$ por lo que se rechaza la H0: La gastronomía típica no es parte de la identidad cultural de los habitantes de Pelileo. y se acepta la HI: La gastronomía típica si es parte de la identidad cultural de los habitantes de Pelileo.

\section{Tabla 3}

Prueba de chi-cuadrado

\begin{tabular}{cccc}
\hline & Valor & gl & Sig. asintótica (bilateral) \\
\hline Chi-cuadrado de Pearson & $368,008^{\mathrm{a}}$ & 64 &, 000 \\
Razón de verosimilitudes & 261,435 & 64 &, 000 \\
Asociación lineal por lineal & 19,863 & 1 &, 000 \\
N de casos válidos & 383 & & \\
\hline
\end{tabular}

Fuente: Llerena (2021)

\section{Tabla 4}

Dimensiones de diseño de un instrumento para medir identidad cultural indígena, caso de estudio sobre la nacionalidad Amazónica"

\begin{tabular}{lll}
\hline \multicolumn{1}{c}{ Dimensiones } & Preguntas & \multicolumn{1}{c}{ Definición } \\
\hline Cognitiva & $(1,2,3)$ & $\begin{array}{l}\text { Definición del sí mismo y su pertenencia a un grupo social que } \\
\text { permite a los individuos agruparse por características y } \\
\text { diferenciarse de otros (Creencias, los valores culturales o la propia } \\
\text { experiencia) }\end{array}$ \\
Evaluativa & $(4,5)$ & $\begin{array}{l}\text { Pertenencia que un individuo tiene a un grupo con el orgullo que } \\
\text { siente de pertenecer a él. Utilización de adjetivos positivos y } \\
\text { negativos }\end{array}$ \\
Afectiva & $\begin{array}{l}\text { Se refiere a la parte emocional e indica que tan cerca se encuentra } \\
\text { la identificación del individuo con el grupo }\end{array}$ \\
Comportamental & $(8,9,10)$ & $\begin{array}{l}\text { Hace referencia a la participación o a su intención de pertenecer al } \\
\text { grupo }\end{array}$
\end{tabular}

Fuente: Pertegal-Felices et al., (2020)

\section{Variables respuesta o resultados alcanzados}

Se obtuvieron el resultado de las 383 encuestas que fueron elaboradas en la plataforma Google form, el instrumento fue aplicado a una población mayor de 15 años de las 
parroquias de Pelileo, por medio de redes sociales para evitar la propagación del Covid19. En la tabla 5 se realiza un análisis e interpretación de resultados de la encuesta aplicada a una muestra de 383 habitantes del cantón Pelileo.

\section{Resultados}

\section{Tabla 5}

Plato Típico predominante de cada parroquia de Pelileo

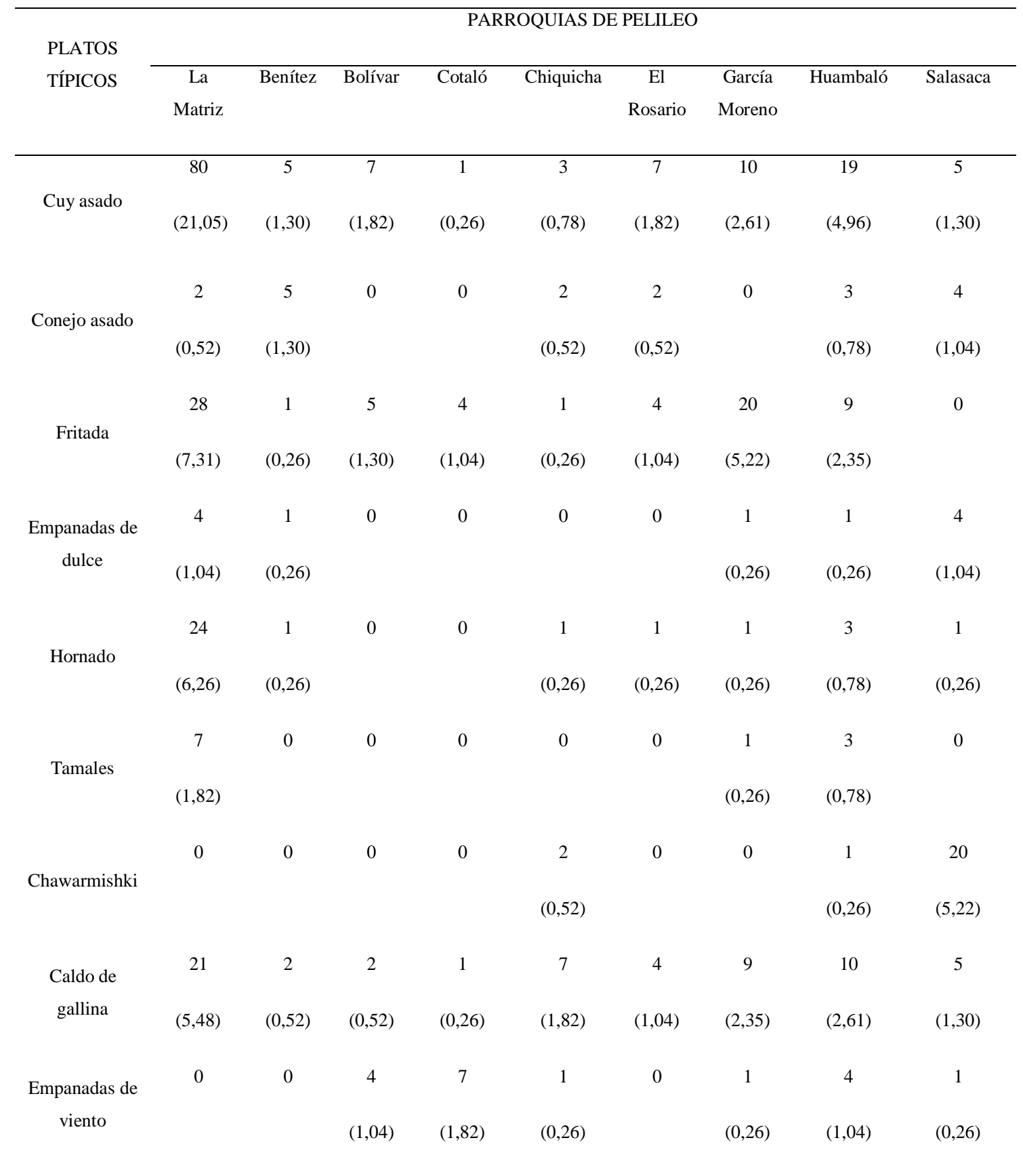

Fuente: Llerena (2021) 


\section{Figura 2}

Plato típico representativo del cantón Pelileo

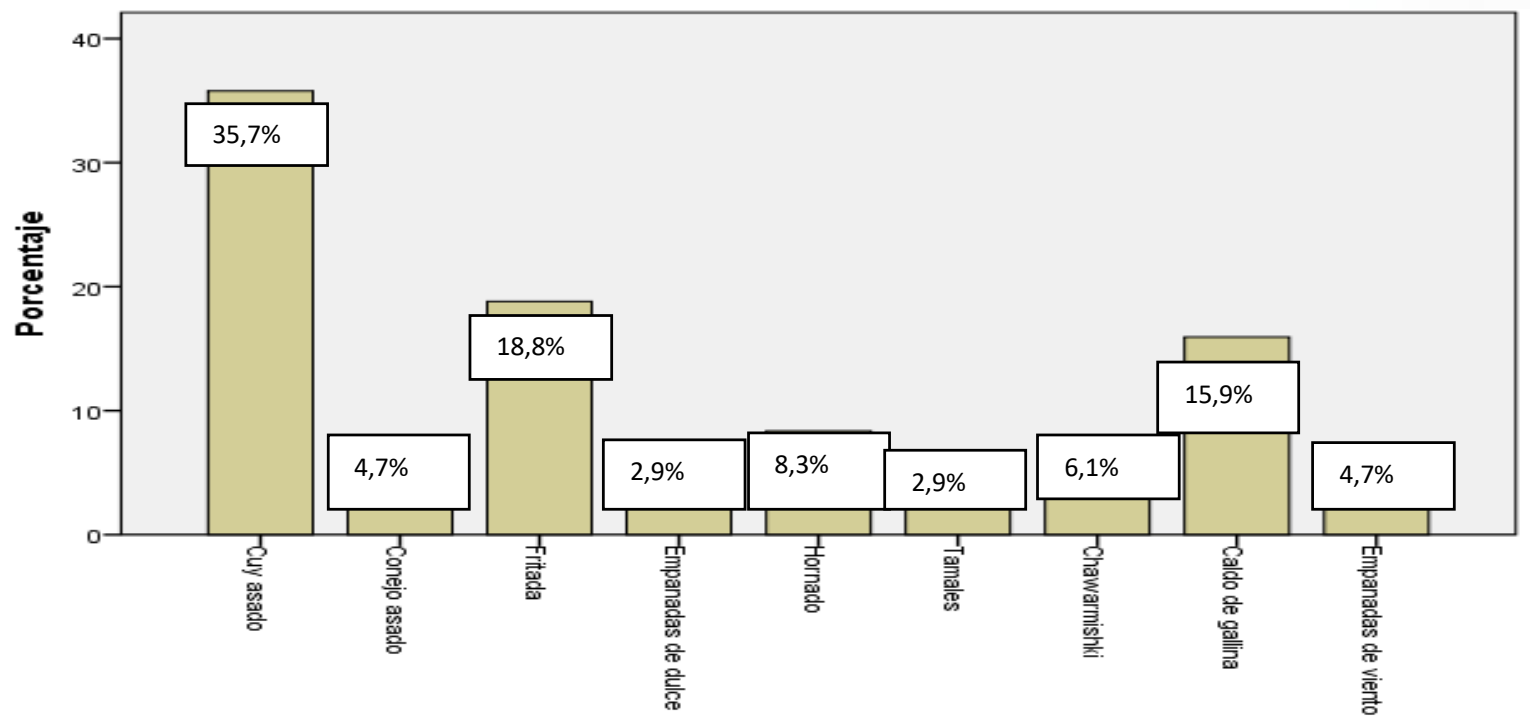

Fuente: Llerena (2021)

Análisis

De las 383 personas encuestadas en el cantón Pelileo que representan el 100\%, en la parroquia la Matriz 80 personas se identifican con el cuy asado que corresponde al $21,05 \%, 2$ personas responden a conejo asado que equivale al 0,52\%, 28 personas elijen fritada que representa el 7,31\%, 4 personas seleccionan empanadas de dulce que corresponde al 1,04\%, 24 personas se identifican con el hornado que corresponde al $6,26 \%, 7$ personas que representan $1,82 \%$ se identifican con tamales y 21 personas que equivale al $5,48 \%$ se identifican con caldo de gallina.

\section{Interpretación de resultados}

Se evidencia que el plato típico principal en el Cantón Pelileo, que identifica a sus habitantes es el cuy asado con el $35,7 \%$, seguido de fritada con un $18,8 \%$ y finalmente el caldo de gallina con 15,9\%. Estas preparaciones típicas se consideran como parte de la identidad cultural gastronómica del cantón Pelileo.

Preguntas de la escala de Likert de la 1-10

Los datos fueron tabulados y procesados en la escala de Likert, de la cual se obtuvo la media aritmética de los factores individuales y globales por medio de las 4 dimensiones de diseño de un instrumento para medir identidad cultural, presentadas en la siguiente Tabla 6, según el rango que corresponde. 


\section{Tabla 6}

Escala de Likert y sus consideraciones

\begin{tabular}{cc}
\hline ESCALA DE LIKERT & CONSIDERACIÓN \\
\hline 1 & Totalmente en desacuerdo \\
2 & En desacuerdo \\
3 & Indiferente \\
4 & De acuerdo \\
5 & Totalmente de acuerdo \\
\hline
\end{tabular}

Fuente: Llerena (2021)

\section{Resultados}

\section{Tabla 7}

Dimensiones de diseño de un instrumento para medir la identidad cultural

\begin{tabular}{ccc}
\hline DIMENSIONES & PROMEDIO & PORCENTAJE \\
\hline Dimensión cognitiva & 3,55 & 18,55 \\
Dimensión evaluativa & 3,57 & 18,64 \\
Dimensión afectiva & 3,80 & 19,84 \\
Dimensión comportamental & 3,58 & 18,68 \\
\hline
\end{tabular}

Fuente: Llerena (2021)

\section{Figura 3}

Resultado de las dimensiones de diseño de un instrumento para medir identidad cultural

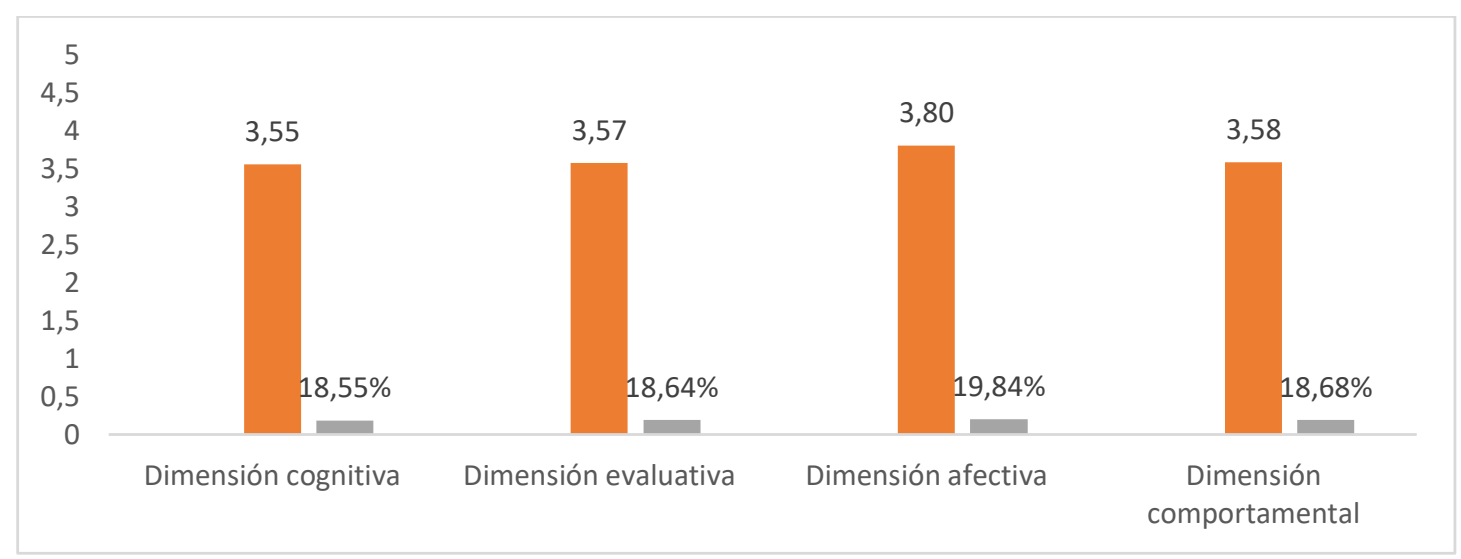

Fuente: Llerena (2021) 
Análisis

Según los datos obtenidos, en lo que corresponde a la dimensión cognitiva representa un promedio de 3,55 que equivale al 18,55\% de los habitantes del cantón Pelileo que tienen una percepción indiferente ante la información de la comida típica que los identifica y no tienen una identidad cultural clara de sí mismo y mucho menos diferenciarse de otros grupos.

Dentro de la dimensión evaluativa muestra un promedio de 3,57 equivalente al 18,64\% de los habitantes del cantón Pelileo que presentan una percepción indiferente sobre sus gustos y preferencias de la comida típica al no sentir pertenencia al tradicionalismo que se desenvuelve de su lugar de origen.

La dimensión afectiva señala un promedio de 3,80 que corresponde al 19,84\% de los habitantes del cantón Pelileo que presentan una percepción indiferente al no tener en alta estima la comida típica como valor cultural que han sido trasmitidas de generación en generación.

En la dimensión comportamental se visualiza un promedio de 3,58 equivalente a $18,68 \%$ de los habitantes del cantón Pelileo que presentan una percepción indiferente al no darle importancia a la comida típica como parte fundamental para el desarrollo turístico del cantón y en consecuencia la poca participación de la población para incentivar esta área turística.

\section{Interpretación de resultados}

El mayor número de habitantes del cantón Pelileo mostró una predilección sobre la dimensión afectiva con un promedio de 3,80 que corresponde al 19,84\% demostrando que las emociones se sobreponen a otros aspectos más lógicos, por ende, se debe dar mayor relevancia a informar por medios factibles la comida típica representativa.

\section{Conclusiones}

- La gastronomía típica es parte de la identidad cultural de los pobladores de San Pedro de Pelileo, ya que es considerada un patrimonio importante para el desarrollo del turismo donde una preparación típica genera una experiencia sensorial única y genera un vínculo con la gastronomía de la región.

- A través de la ficha de registro del INPC se identificaron 179 establecimientos con los platos típicos más icónicos de Pelileo tales como: cuy asado conejo a la parrilla papas fritas pan dulce al horno tamales chawarmishki caldo de pollo y papel de arroz mezclado con viento.

- Los principales platos típicos del cantón Pelileo, que identifican a sus habitantes son: cuy asado con el $35,7 \%$, seguido de fritada con un $18,8 \%$ y finalmente el 
caldo de gallina con 15,9\%. Mostrando que la identidad cultural del habitante del cantón Pelileo pertenece a la dimensión afectiva con un promedio de 3,80 que corresponde al 19,84\% esto demuestra que las emociones se sobreponen a otros aspectos más lógicos.

\section{Referencias bibliográficas}

Bohórquez, A., López, N. \& Gómez, A. (2016). Estrategia para el reconocimiento de la identidad cultural y el sentido de pertenencia de inmigrantes latinoamericanos. El Ágora USB, 16(2), 383-392.

Bombón, S. (2017). La gastronomía típica como un factor de desarrollo turístico del cantón Tisaleo provincia de Tungurahua (Tesis de Maestría). Universidad Técnica de Ambato

Bugallo, B. (2020). Creaciones gastronómicas y su protección legal, con especial referencia a la propiedad intelectual. Revista de derecho 1 (38), 3-8

Bravo, O., \& Vallejo, J. (2018). La Evolución De La Gastronomía En La Academia A Través Del Tiempo. Revista Turydes: Turismo y Desarrollo, 11(24), 1-12.

Castellanos, B. (2017). El uso de los métodos deductivo e inductivo para aumentar la eficiencia del procesamiento de adquisición de evidencias digitales. Cuadernos de contabilidad, 18(46).

Fernández, A. \& Miret, F. (2019). La gastronomía como recurso de la identidad ancestral: el caso de la Parroquia Chuquiribamba, Loja, Ecuador. Revista Amazónica Ciencia y Tecnología, 8(2), 126-135.

Garza, L. \& Llanes, A. (2015). Modelo pedagógico para desarrollar la identidad cultural. Humanidades Médicas, 15(3), 562-581.

Lind, D., Marchal, W., Wathen, S., Obón León, M. \& León Cárdenas, J. (2012). Estadística aplicada a los negocios y la economía. México: McGrawHill/Interamericana Editores.

Lardellier, P. (2015). ¿Ritualidad versus modernidad...? Ritos, identidad cultural y globalización. Revista Mad. Revista del Magíster en Análisis Sistémico Aplicado a la Sociedad, (33), 18-28.

Llerena, K. (2021). Gastronomía típica como identidad cultural. [Tesis de maestría, Universidad Técnica de Ambato]. https://repositorio.uta.edu.ec/bitstream/123456789/32997/1/180402398\%20KAR INA\%20PATRICIA\%20LLERENA\%200\%C3\%91ATE.pdf 
Martínez, N. (2015). Identidad cultural y educación. Recuperado de http://redicces.org.sv/jspui/bitstream/10972/2055/1/3.\%20Identidad\%20cultural $\% 20 y \% 20$ educacion.pdf

Mogollón, J., DiClemente, E., \& Guzmán, T. (2015). El turismo gastronómico como experiencia cultural. El caso práctico de la ciudad de Cáceres (España). Boletín de la Asociación de geógrafos españoles, 68 (1), 407-427.

Moposita, L. (2016). Fortalecimiento histórico cultural de la gastronomía del cantón San Pedro de Pelileo. (Tesis de Maestría). Tungurahua.

Navarrete, Z. (2015). ¿Otra vez la identidad?: Un concepto necesario pero imposible. Revista mexicana de investigación educativa, 20(65), 461-479.

Saltos, M., \& Raymond, V. (2018). Televisión Comunitaria: factores de éxito para el crecimiento en Ecuador. Killkana sociales: Revista de Investigación Científica, 2(1), 27-32.

Pérez, J., Ramos, M., \& Tapia, S. (2017). Riesgos psicosociales y la seguridad industrial en las lavanderías textiles del Cantón Pelileo. Revista de la SEECI, (43), 135-149.

Pertegal-Felices, M., Espín-León, A. \& Jimeno-Morenilla, A. (2020). Diseño de un instrumento para medir identidad cultural indígena: caso de estudio sobre la nacionalidad amazónica Waorani. Revista de Estudios Sociales, (71), 51-61.

\section{Ciencia}


El artículo que se publica es de exclusiva responsabilidad de los autores y no necesariamente reflejan el pensamiento de la Revista Conciencia Digital.

\section{Ciencia \\ LDigital}

El artículo queda en propiedad de la revista y, por tanto, su publicación parcial y/o total en otro medio tiene que ser autorizado por el director de la Revista Conciencia Digital.
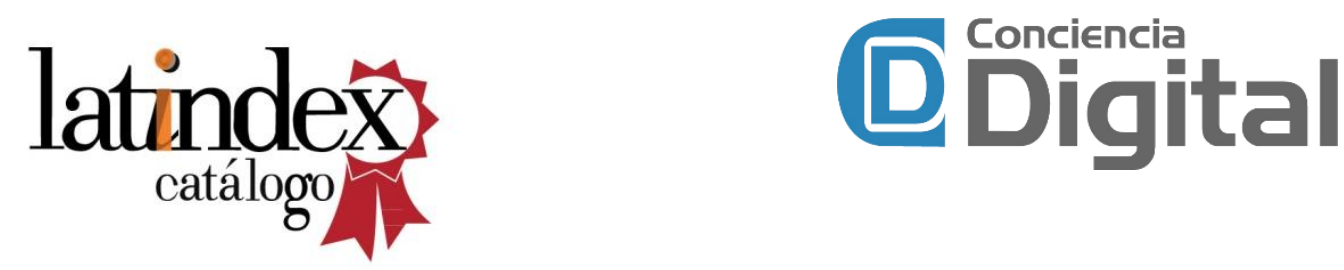

Indexaciones

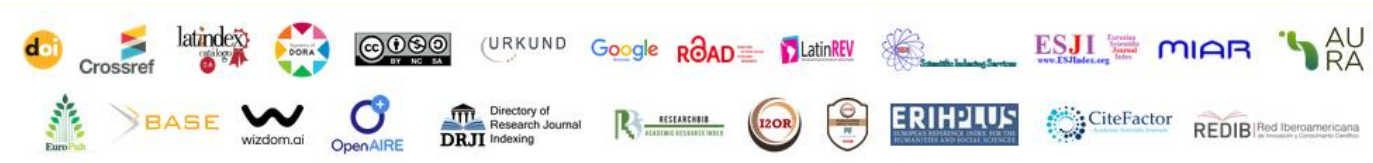

